Japan. J. Med. Sci. Biol., 24, 313-321, 1971

\title{
LIMITS IN TUBERCULOSIS CHEMOTHERAPY AS REVEALED BY EXPERIMENTAL STUDY IN MICE
}

\author{
KOOMI KANAI* AND EIKO KONDO \\ Department of Tuberculosis, National Institute of Health, \\ Shinagawa-ku, Tokyo 141, Japan
}

(Received: April 4th, 1971)

\begin{abstract}
SUMMARY : Viable forms of tubercle bacillus, even when they are essentially drug-sensitive, can persist in mouse tissue under the treatment with antituberculous drugs. This peculiar phenomenon characteristics of the chronic stage of infection is called "microbial persistence". To explain the underlying mechanism, some model experiments were conducted. Infection with resting bacilli was attained in mice with a streptomycin-dependent strain having completed residual growth on the medium lacking the antibiotic. Isoniazid administration failed to eliminate such resting bacilli from the mouse tissue. An additional experiment showed that the challenge inoculum whose multiplication was inhibited by vaccine-induced immunity did not respond satisfactorily to the mycobactericidal action of isoniazid. These observations and others led us to a view that microbial persistence results from the insensitiveness to chemotherapeutics of the bacilli in a resting condition under the pressure of infection immunity.
\end{abstract}

\section{INTRODUCTION}

McCune and Tompsett (1956), McDermott (1958) and McCune et al. (1966) reported that viable forms of tubercle bacilli persisted, if not multiplied, in the tissue of mice despite prolonged treatment with antituberculous chemotherapeutics. They added that this occurred though the bacilli remained essentially drug-sensitive. These findings appear to explain the clinical experience that active tuberculosis can be brought to a resting state under control by chemotherapy, and also explain the epidemiological situation that tuberculosis patients are now shifting from younger generation to older one mostly as relapse cases due to reactivation of the primary infection. A question is raised here why eradication of causative microorganisms is difficult in tuberculous infection even with such powerful antituberculous drugs as isoniazid. To answer this question, Kanai (1966) has already reported some preliminary study in experimental murine tuberculosis, and has drawn a tentative conclusion that chemotherapeutics would not act against tubercle bacilli being harbored in the tissue in a resting or non-multiplying state. The present paper is to confirm and extend the previous observations.

*The present address: Yamanashi Prefectural Institute for Public Health.

The work described in this paper has been published in Japanese in two divided parts (Kekkaku, 44, 379, 1969 and Kekkaku, 45, 159, 1970).

金井 興美・近藤 塋子 (国立予防衛生研究所 結核部) 


\section{MATERIALS AND METHODS}

Experimental animals: Mice were of the commercially available dd strain, male, weighing $20 \mathrm{~g}$ on the average. They were housed, 10 per box, on wood shavings in metal boxes with wire-grid tops, and were fed with pellet diet and water.

Microorganisms : A kanamycin-resistant strain H37RvKM-R, a streptomycinresistant strain H2SM-R, and a streptomycin-dependent strain H2SM-D designated as $18 \mathrm{~b}$, each being a one-step mutant strain of M. tuberculosis, were the microorganisms employed. All these strains have been maintained on glycerol-egg slants (Ogawa type) in this Department. The streptomycin-dependent strain was first isolated by Hashimoto and some biological properties were described by him (Hashimoto, 1955a, b). This strain requires streptomycin in concentration higher than $40 \mu \mathrm{g}$ per $\mathrm{ml}$ for its satisfactory growth on glycerol-egg medium or on Sauton synthetic liquid medium. H37 RvKM-R and H2SM-R are resistant to the respective antibiotic of at least $100 \mu \mathrm{g}$ per $\mathrm{ml}$.

Preparation of vaccine and challenge suspension : Bacillary suspension was prepared from pellicle growth on Sauton medium. The growth was harvested on a funnel and then dried by pressing between layered folds of absorbent filter paper. A weighed portion of thus prepared bacillary mass was put into a round flask of 300 $\mathrm{ml}$ capacity containing 60 beads. Finely dispersed bacillary suspension was prepared by rotating the flask manually with occasional addition of distilled water as suspending medium. The amount of water was so adjusted that desired concentrations (mg per $\mathrm{ml}$ ) of the bacilli might be obtained.

Induction of a resting state of viable vaccine: The streptomycin-dependent strain was grown on the surface of Sauton medium containing dihydrostreptomycin sulfate in $100 \mu \mathrm{g}$ per $\mathrm{ml}$, and then a loopful mass of this growth was transferred onto antibiotic-lacking fresh Sauton medium. This inoculum usually made a residual growth to some extent, and then ceased to develop. After this culture condition had been confirmed vaccines were prepared by the above described method to be used as a resting viable inoculum. The time necessary for such streptomycin-starvation is usually one to two weeks.

Enumeration of viable units from vaccinated and infected organs: Mice were sacrifficed by cervical dislocation to remove aseptically the spleen and the lungs. The organs were weighed, washed with distilled water, and placed into a mortar or a glass-homogenizer. Each tissue homogenate was so prepared as to make a 1: 10 dilution in distilled water, and it was passed through a funnel with a sheet of gauze. When required, two or three serial ten-fold dilutions were prepared from this filtrate, $0.1 \mathrm{ml}$ amounts of each dilution were inoculated on to three slants of glycerolegg medium. The number of colonies developed after incubation at $37 \mathrm{C}$ for 5 weeks allowed calculation of the numbers of viable units present in the tissue. H2SM-R, H2SM-D or H37RvKM-R was conveniently enumerated on the medium containing streptomycin or kanamycin in $100 \mu \mathrm{g}$ per $\mathrm{ml}$; the antibiotics prevented bacterial contamination and made differential isolation possible.

Chemotherapeutics: Dihydrostreptomycin sulfate or isoniazid was administered intraperitoneally to mice. When streptomycin sulfate or kanamycin sulfate was incorporated into glycerol-egg medium, the amount to be added was so adjusted with a consideration that the final potency in the completed medium is reduced to one half the original in the former and to one tenth in the latter. 


\section{EXPERIMENTAL RESULTS \\ Isoniazid-treatment of Experimental Mouse Tuberculosis with Different Effects on the Fate of Tissue Viable Counts in the Lung and Spleen}

Virulent tubercle bacilli can multiply more freely and persist on a higher level of viable counts in the lung than in the spleen of mice (Kanai and Kondo, 1968; North and Mackaness, 1970). This phenomenon would be understood as the tissue differnece in the development and operation of acquired immunity to tuberculous infection. With this information in mind, the first experiment was conducted to compare the effect of isoniazid-treatment on the fate of bacilli in the two organs.

Forty mice were intravenously infected with $0.005 \mathrm{mg}$ of H37RvKM-R strain. One day and one week later, two mice were sampled at random for enumeration of viable units of the lung and spleen with kanamycin-containing slants. The remaining animals were then divided into two groups of equal half, one of which was treated by daily administration of $0.5 \mathrm{mg}$ isoniazid, 6 times each week, and continued until the termination of experiment. During this period, the enumeration was done with 3 animals from each experimental group. The results are shown in Fig. 1 . In the lung of the untreated mice, the bacilli multiplied showing the two log increase in viable counts for the first three weeks, and then persisted maintaining the same level of the counts. The therapeutic effect of isoniazid was marked modifying the fate of the bacilli in the lung. After one-week administration of the drug, the count decreased down to one hundredth of that in the untreated group. Continuous administration of isoniazid could not, however, bring about further elimination of the bacilli, but it worked only to maintain the lowered level of viable.counts at least during the later 4 weeks. The persistence of viable forms of tubercle bacilli under chemotherapy was revealed in this way. Nevertheless, because of the bacillary
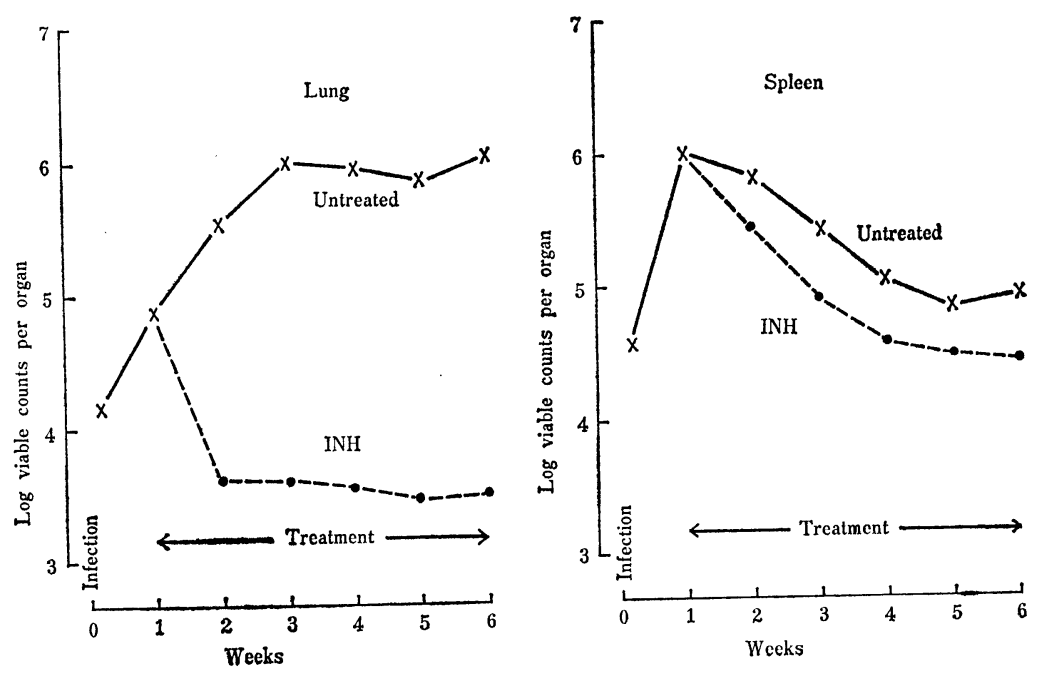

Fig. 1 Different effects of isoniazid treatment on the fate of infecting bacilli in the spleen and lung. 
multiplication for the first three weeks in the untreated group, the ratio of viable counts between the two groups became almost three log level finally. In contrast to this situation in the lung, the bacilli in the spleen multiplied only for the first week and then declined in number. Isoniazid administration could, however, accelerate little this natural course of decline, the difference of viable counts between the two groups being rather small.

\section{Influence of BCG Vaccination on the Efficacy of Isoniazid Treatment Against Subsequent Challenge Infection}

Forty-four mice were divided into 2 groups of equal half. The animals of one group were vaccinated intravenously with $0.1 \mathrm{mg}$ of BCG. Four weeks later, both groups were challenged intravenously with $0.01 \mathrm{mg}$ H2SM-R, and further divided into two subgroups of equal 11. From the 3rd day of infection, each of the vaccinated or unvaccinated subgroups were treated by daily administration of $0.5 \mathrm{mg}$ isoniazid, six times each week. With 3 mice on the 3rd day of infection from each subgroup and then with 4 animals one and two weeks after infection, enumeration of spleen viable units was conducted on streptomycin-containing glycerol-egg slants. The results are shown in Fig. 2. The figure shows that BCG vaccination inhibited the multiplication of challenge inoculum in the spleen. The difference in viable count between the untreated and treated subgroups indicates that the isoniazid administration was less effective against such inhibited course of infection than against the multiplying bacilli in the unvaccinated animals.
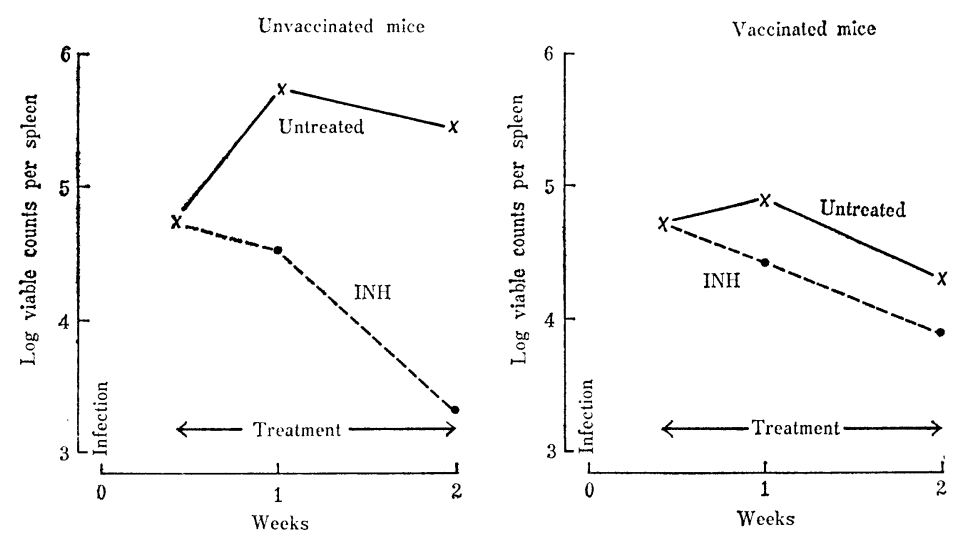

Fig. 2 Effect of BCG-vaccination on the isoniazid treatment against subsequent challenge infection with virulent tubercle bacilli.

\section{Isoniazid-treatment of the Mice Infected with Resting Tubercle Bacilli Obtained in Vitro by Antibiotic-starvation of a Streptomycin-dependent Strain}

Eighty mice were infected intravenously with $0.05 \mathrm{mg}$ of the antibiotic-starved bacilli of a streptomycin-dependent strain (18b) of tubercle bacilli prepared by the method as described before. One day and one week after infection, four mice were 

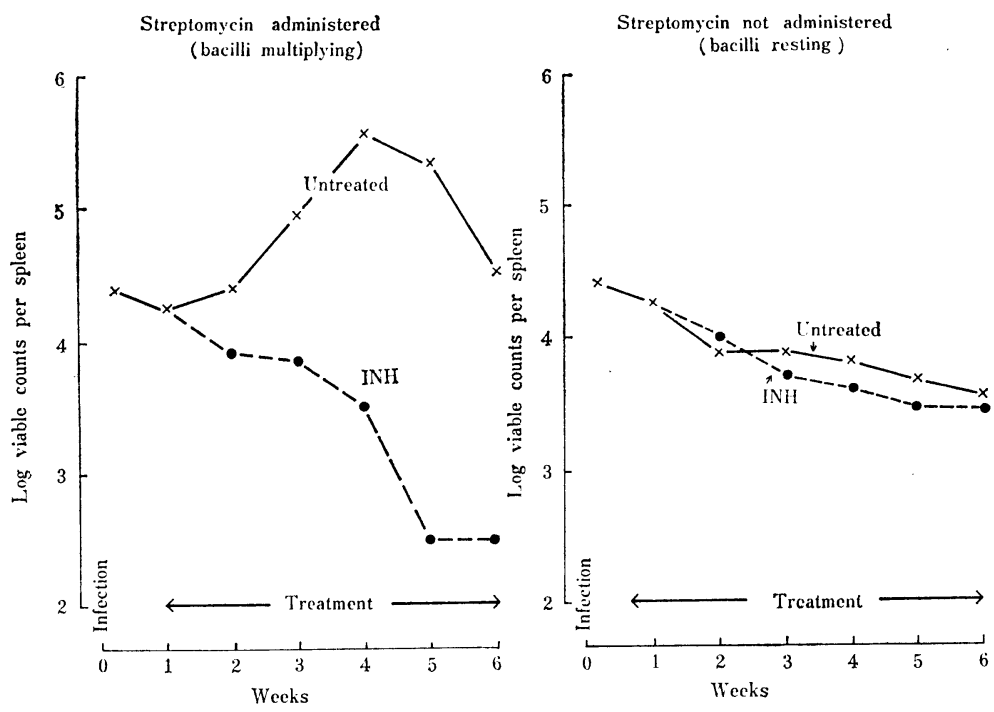

Fig. 3 Failure of isoniazid treatment against infection with nonmultiplying tubercle bacilli (Use of the antibiotic-starved bacilli of a streptomycin-dependent strain).

sacrificed for enumeration of spleen viable units using streptomycin-containing glycerolegg slants. Then, the remaining animals were divided into two groups of equal half. Daily administration of $10 \mathrm{mg}$ streptomycin was conducted only to the mice of one group. At the same time, both groups were divided into two subgroups, one of which was to be treated by daily administration of $0.5 \mathrm{mg}$ isoniazid. The treatment was done 6 times a week and continued until the termination of experiment. During the experimental period, three mice were sampled weekly from each subgroup to follow the fate of infecting bacilli in the spleen. The results are shown in Fig. 3.

When bacillary multiplication was supported by streptomycin administration, isoniazid exerted a marked therapeutic effect reducing tissue viable counts down to one thousandth of the untreated case at the miximal difference. On the other hand, when streptomycin was not given to the animals, the bacilli in the tissue declined very slowly to one tenth of the starting viable counts in six weeks. In this situation, isoniazid-treatment could not exert any influence on such fate of bacilli.

\section{Isoniazid Treatment against Mixed Infection with Multiplying and Resting Tubercle Bacilli}

To confirm the preceding observation in a different experimental system, the next experiment was conducted. Eighty mice were infected with the combined inoculum of streptomycin-starved bacilli $(0.01 \mathrm{mg})$ of $18 \mathrm{~b}$ strain and H37HvKM-R $(0.005 \mathrm{mg})$. One day and one week after infection, four mice were sacrificed for enumeration of spleen viable units by the simultaneous use of two kinds of glycerolegg medium containing streptomycin or kanamycin. Thus, differential isolation of the two strains was possible. The remaining animals were then divided into two groups 

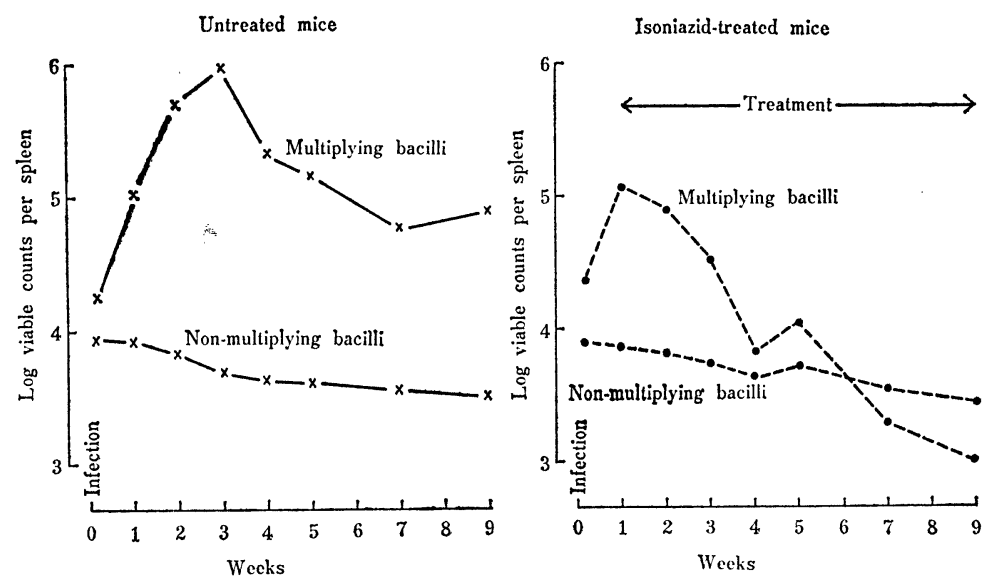

Fig. 4. Isoniazid treatment against infection with a mixed inoculum of non-multiplying (the streptomycin-dependent strain) and multiplying (kanamycin-resistant strain) tubercle bacilli.

of equal half, and the mice of one group were treated by daily administration of 0.5 $\mathrm{mg}$ isoniazid. With one week intervals, four animals were sacrificed from each group to enumerate spleen viable counts as before. The results are shown in Fig. 4. From this figure, it will be seen that isoniazid was effective only against the multiplying bacilli also when they coexisted with the non-multiplying bacilli.

\section{Relapse and Chemotherapy in Experimental Mouse Tuberculosis}

One hundred and fifty mice were intravenously infected with $0.002 \mathrm{mg}$ of the antibiotic-starved cells of $18 \mathrm{~b}$ strain. The next day of infection, four animals were sacrificed to be examined of the initial distribution of the infection inoculum in the spleen and lung, and the remaining animals were divided into two groups of equal half (A and B). To the mice of Group B, streptomycin was administered daily in the dose of $10 \mathrm{mg}$ for the first two weeks to support the multiplication of the inoculum. With two week intervals, enumeration of viable nuits in the both organs was made with 4 animals from each group for ten weeks. Then, both groups were divided into two subgroups of similar numbers of mice. One of the subgroups was treated by daily administration of $10 \mathrm{mg}$ streptomycin for 4 weeks and the other group by combined administration of $10 \mathrm{mg}$ streptomycin and $0.2 \mathrm{mg}$ isoniazid. Hereafter, enumeration of tissue viable counts was done as before with two week intervals with 4 animals from each subgroup.

The change of viable counts in Group A during the whole experimental period is demonstrated in Fig. 5. Without the supply of streptomycin, viable counts in the spleen decreased gradually down to one tenth of the initial count at the 10th week. However, when streptomycin administration was started at this time, viable units began to increase reaching the initial level as early as in two weeks and then the counts tended to decrease even under the continuous administration of streptomycin. When isoniazid was administered in combination with streptomycin, viable counts continued to decrease still after the 10th week of infection and finally entered into 


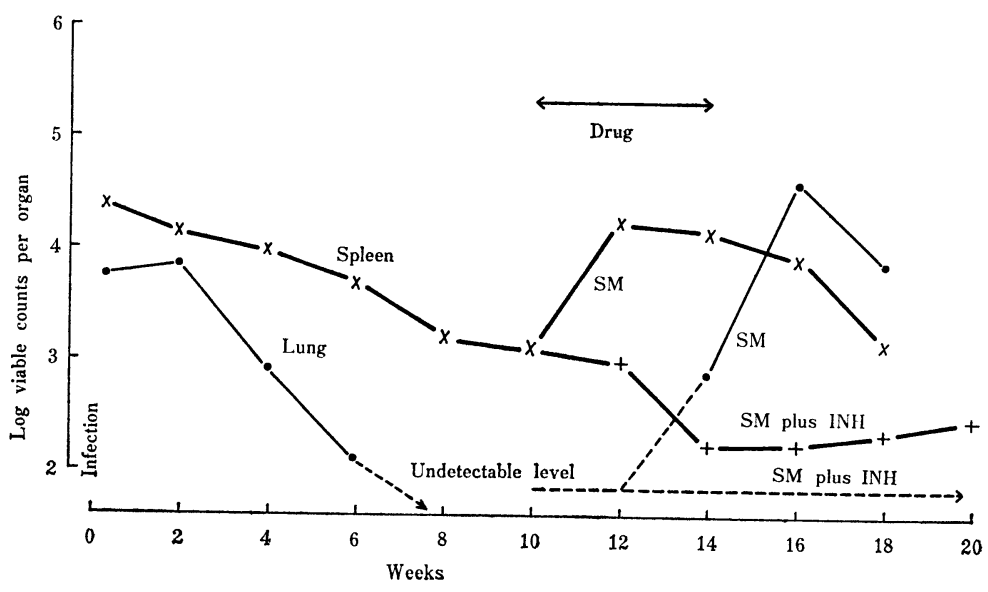

Fig. 5. Resting primary infection in mice with a streptomycindependent strain of tubercle bacilli and later treatment with isoniazid under the streptomycin-induced activation.

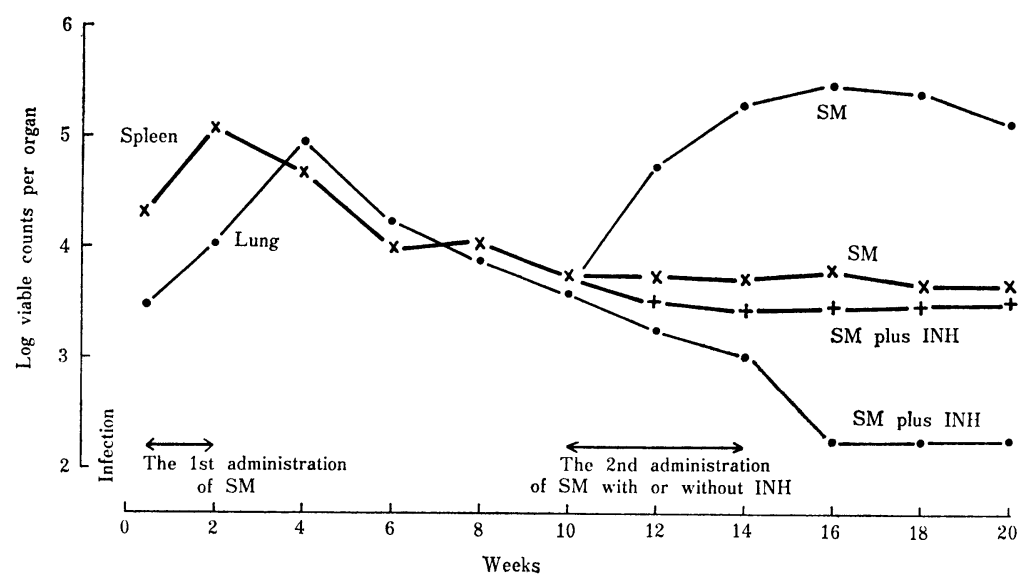

Fig. 6. Active primary infection in mice with a streptomycinde pendent strain of tubercle bacilli and later administration with isoniazid under streptomycin-induced reactivation.

the stage of microbial persistence at the 14 th week. On the other hand, the bacilli in the lung showed a fairly rapid decrease in viable count, and no bacilli was recovered at the 8 th, 10 th and 12 th week. However, when streptomycin was administered starting from the 10th week, the bacilli became detectable again by culture, even to a higher level of viable counts sixweeks later. This reappearance of cultivable bacilli was completely inhibited by simultaneous administration of isoniazid.

Unlike Group A, the animals of Group B were subjected to daily administration of $10 \mathrm{mg}$ streptomycin for the first two weeks of infection. As the result, the bacilli multiplied in the spleen and lung to some extent. This procedure was to 
develop infection immunity by the bacilli stimulated to multiplication. They showed a peak of tissue viable counts in 2 to 4 weeks and then decreased until the 10th week of infection (Fig. 6). The readministration of streptomycin for 4 weeks was started at this time, which was successful in supporting again the bacillary multiplication in the lung, but failed to do so in the spleen allowing microbial persistence there until the 20th week of infection. The figure shows that combined administration of isoniazid was effective against the multiplying bacilli in the lung, but not against the persisting bacilli in the spleen.

\section{DISCUSSION}

It has generally been accepted that chemotherapeutics are effective especially against microorganisms under multiplication. In vitro experiments can reveal the fact that bacterial cells are most sensitive to chemotherapeutics when they are in the logarithmic phase of growth (Dubos, 1945). This is also true with tubercle bacilli in connection with antituberculous drugs (Kanai and Yanagisawa, 1955a). On the other hand, few literatures have been available which demonstrate the relation between drug sensitivity and the growth phase of tubercle bacilli in vivo. It appears that if this could be clarified, we would come to an understanding as to why viable forms of tubercle bacilli can persist in the chronic phase of infection despite the continuous administration of chemotherapeutics.

The use of the streptomycin-dependent strain of tubercle bacilli was advantageous for animal experiments for the above purpose. This strain, when deprived of streptomycin, can survive without multiplication for a fairly prolonged period of time in vivo as well as in vitro (Kanai, 1966). Using this strain, the present study proved that such surviving bacilli do not respond to the strong mycobactericidal action of isoniazid in vivo. This is an evidence favouring the view that the microbial persistence under chemotherapy is due to the drug-insensitive condition of the nonmultiplying bacilli. Meanwhile, we must raise a question whether the above observation is specific only to this particular experimental system employed or is can be taken as a general principle.

In the later stage of infection, tubercle bacilli can survive in the host maintaining almost the same level of viable counts for a long period (Kanai and Yanagisawa, 1955b) (McCune and Tompsett, 1956). This is one of the characteristic features of chronic infection. The host-parasite equilibrium as such is "static" in nature rather than "dynamic" as evidenced by Rees and Hart (1961) and Kanai (1966). In other words, tissue viable counts in this stadium mean the continuous presence of the same bacterial cells which are viable but not in multiplication, and do not mean a balanced number between multiplication and destruction. And it can be added that the equilibrium will be based upon the competition between bacillary "virulence" and acquired host resistance. Our results as shown in Fig. 2 and Fig. 6 are the good evidence to prove that acquired immunity induced by vaccination or infection suppresses the multiplication of challenge inoculum, consequently reducing their sensitivity to isoniazid. In this sense, immunity is intimately interfering with the drug-parasite relationship. In fact, paradoxically as it may sound, defense mechanism of the host established by immunological processes is a limiting factor to the action of chemotherapeutic agents. 
The peculiar observation as this had already been obtained by Egashira, Ogawa and Sakurabayashi (1953) in tuberculous guinea pigs, in which BCG-prevaccination and isoniazid-administration appeared to have worked competitively.

\section{ACKNOWLEDGEMENT}

The present authors have been encouraged by Dr. M. Naito, Professor of Internal Medicine in the Chest Disease Institute of Kyoto University, for the performance of this study. We express our deep appreciation for his kind guidance.

\section{REFERENCES}

Dsubos, R. J. (1945): The Bacterial Cell, Harvard University Press, Massachusetts, $460 \mathrm{p}$. EgashiRA, Y., OGAWA, H. AND SAKURABAYASHI, T. (1953): Experimental studies on chemotherapy of tuberculosis (Report VII). Comparative experiments on the therapeutic effect of isoniazid and streptomycin upon tuberculosis in BCC-immunized and non-immunized guinea pigs. Trans. Soc. Pathol. Jap., Edit. Region., 42, 290-291 (text in Japanese).

HASHIMOTO, T. (1955a): Experimental studies on the mechanism of infection and immunity in tuberbulosis from the analytical standpoint of streptomycin-dependent tubercle bacilli. I. Isolation and biological characteristics of a streptomycin-dependent mutant, and effect of streptomycin administration on its pathogenicity in guinea pigs. Kekkaku, 30, 4-8 (text in Japanese).

HASHimoto, T. (1955b): Experimental studies on the mechanism of infection and immunity in tuberculosis from the analytical standpoint of streptomycin-dependent tubercle bacilli. II. Studies on the virulence of back mutants from streptomycin-dependent tubercle bacilli. Kekkaku, 30, 237-241 (text in Japanese).

KANAI, K. (1966): Experimental studies on host-parasite equilibrium in tuberculous infection, in relation to vaccination and chemotherapy. Japan. J. Med. Sci. Biol., 19, 181-199.

KANAI, K. AND KONDO, E. (1968): Studies on lysosomal response to tuberculous infection in mice. Japan. J. Med. Sci. Biol., 21, 415-422.

KANAI, K. AND YANAGISAWA, K. (1955a): Antibacterial action of streptomycin against tubercle bacilli of various groth phase. Japan. J. Med. Sci. Biol., 8, 63-76.

KANAI, K. AND YANAGISAWA, K. (1955b): Studies on the reinfection in experimental tuberculosis of guinea pigs. Japan. J. Med. Sci. Biol., 8, 115-127.

MCDermotT, W. (1958): Microbial persistence. Yale J. Biol. Med., 30, 257-291.

MCCune, R. M. Jr. AND TOMPSETT, R. (1956): Fate of Mycobacterium tuberculosis in mouse tissues as determined by microbial enumeration technique. I. The persistence of drug-susceptible tubercle bacilli in the tissues despite prolonged antimicrobial therapy. J. Exptl. Med., 104, 737-762.

McCune, R. M., Feldman, F. M., Lambert, H. P. and McDermott, W. (1966): Microbial persistence. I. The capacity of tubercle bacilli to survive sterilization in mouse. J. Exptl. Med., 123, 445-468.

REES, R. J. W. AND HART, D. P. (1961): Analysis of the host-parasite equilibrium in chronic murine tuberculosis by total and viable bacillary counts. Brit. J. Exptl. Pathol., $42,83-88$. 